\title{
The Admid System: Generation of Recombinant Adenoviruses by Tn7-Mediated Transposition in $E$. coli
}

BioTechniques 29:146-154 (July 2000)

\section{Cynthia A. Richards, Chadwick E. Brown, John P. Cogswell and Michael $P$. Weiner}

Glaxo Wellcome, Research

Triangle Park, NC, USA

\section{INTRODUCTION}

Adenoviruses (Ads) are 36-kb, linear, dsDNA viruses that have been widely used for gene therapy, functional genetics, vaccines and protein production $(16,19,20,24,26)$. Most Ad vectors are based on the Ad type 5 (Ad5) viral backbone in which an expression cassette replaces the E1 and/or E3 region. Such E1- ${ }^{-}$E3- viruses are attractive vectors because they: (i) can accept about $8 \mathrm{~kb}$ exogenous DNA, (ii) can be produced at very high titer in cells expressing E1 and (iii) are replication defective. Other attractive features of Ad vectors include high-efficiency infection of a wide range of both dividing and quiescent cell types, high-level gene expression and historical association with mild, self-limiting pathologies in humans $(4,16,19,20,24,26)$. However, for gene therapy applications, recent clinical results suggest the toxicity of high-level recombinant Ad infection has been underestimated (21). The increasing numbers of genes discovered by the human genome project and the expanding field of gene therapy are driving the need for more efficient systems to generate recombinant Ad vectors to facilitate rapid, high-throughput functional and therapeutic analysis.

Because of their complex genome, manipulation of Ad vectors to produce recombinant viruses has been difficult. Traditional methods of making recombinant Ads use homologous recombination between two transfected DNAs in Ad-producing cell lines (4). The homologous recombination method is inefficient, time-consuming and subject to contamination with replication-competent virus. Some newer methods of generating recombinant Ad vectors in yeast and $E$. coli have been reported $(7,8,14,16)$. These methods shift the homologous recombination step from eukaryotic packaging cells into either yeast or E. coli. Another recent method (25) uses rare-cutting, intron-encoded endonucleases to insert transgenes into a plasmid bearing a full-length Ad genome. These newer methods allow the true cloning of the recombinant viral genome and remove the need for clonal selection with repeated rounds of plaque purification. A method using the Cre-loxP system to generate recombinant $\mathrm{Ad}$ by in vitro recombination has also been reported (1). This method does not allow cloning in $E$. coli, but the recombination results in only one molecule that can produce clonal Ads.

A method that has been used to construct recombinant baculoviruses in $E$. coli by Tn7-mediated, site-specific transposition (22) has proven to be very efficient for generating recombinant baculoviruses. We have developed a Tn7-based transposition system for generating recombinant Ads in E. coli. A low copy number adenovirus plasmid (admid) containing a full-length Ad genome with lacZatt $T n 7$ replacing E1 has been constructed. The admid system is easy, rapid and efficient, accommodates DNA inserts up to $5.8 \mathrm{~kb}$ in the current $\mathrm{E}^{-}, \mathrm{E}^{-}$- deleted vectors and generates truly clonal viruses. This system is also easily adapted to allow subcloning into a single universal transfer vector that can be used to transpose genes into any of several different expression or viral vector systems, thus facilitating cost-effective subcloning into Ad, baculoviral and E. coli vectors (M.W., personal communication). 


\section{MATERIALS AND METHODS}

\section{Bacterial Strains, Plasmids, Cell Lines and Viral DNA}

DH10B and DH10Bac competent cells and pFASTBAC1 were obtained from Life Technologies (Rockville, MD, USA). The plasmid pMON7124 (3) was isolated from DH10Bac cells. BJ5183 (13) was provided by D. Hanahan and pGL3-basic was purchased from Promega (Madison, WI, USA). The tetracycline-regulatable promoter (TetOp) and tetracycline-controlled transactivator (tTA) were obtained from $\mathrm{H}$. Bujard (10). P. Fasani-Ghersa (Serono, Geneva, Switzerland) constructed and provided AdtTA. Other reagents include pFOS1 (18), IN340 viral DNA (15), HEK 293 cells (11) and IMR-90 cells (23).

\section{General Methods}

Competent cells were prepared by the $\mathrm{CaCl}_{2}$ method and stored frozen (2). Plasmid minipreps were prepared using Wizard ${ }^{\circledR}$ minipreps (Promega); plasmids larger than $20 \mathrm{~kb}$ were eluted from the minicolumns with distilled water heated to $80^{\circ} \mathrm{C}$. For large-scale isolation, 500-1000-mL cultures were grown in LB medium, and the admids were isolated using plasmid maxi kits following the procedure recommended for low copy number plasmids (Qiagen, Valencia, CA, USA). LB agar was supplemented with $100 \mu \mathrm{g} / \mathrm{mL}$ ampicillin (Ap), $15 \mu \mathrm{g} / \mathrm{mL}$ tetracycline (Tc), 20 $\mu \mathrm{g} / \mathrm{mL}$ chloramphenicol $(\mathrm{Cm}), 300$ $\mu \mathrm{g} / \mathrm{mL}$ Bluo-gal (Life Technologies) and $40 \mu \mathrm{g} / \mathrm{mL}$ isopropylthio- $\beta$-galactoside (IPTG) as indicated. PCR was performed with AmpliTaq ${ }^{\circledR}$ (PE Biosystems, Foster City, CA, USA) using standard techniques (2).

\section{Construction of Admid Homing Vectors and Transfer Vectors}

The admid vector was constructed in a multistep procedure from IN340 viral DNA, bMON14272 and pFOS1. The final admid contains an $\mathrm{E}^{-}, \mathrm{E} 3^{-} \mathrm{Ad}$ genome, the low copy number $\mathrm{F}^{\prime}$ origin and $\mathrm{Cm}$-resistance gene (Figure 1). The lacZatt Tn7 (generated by PCR of DH10Bac DNA with primers priCR1

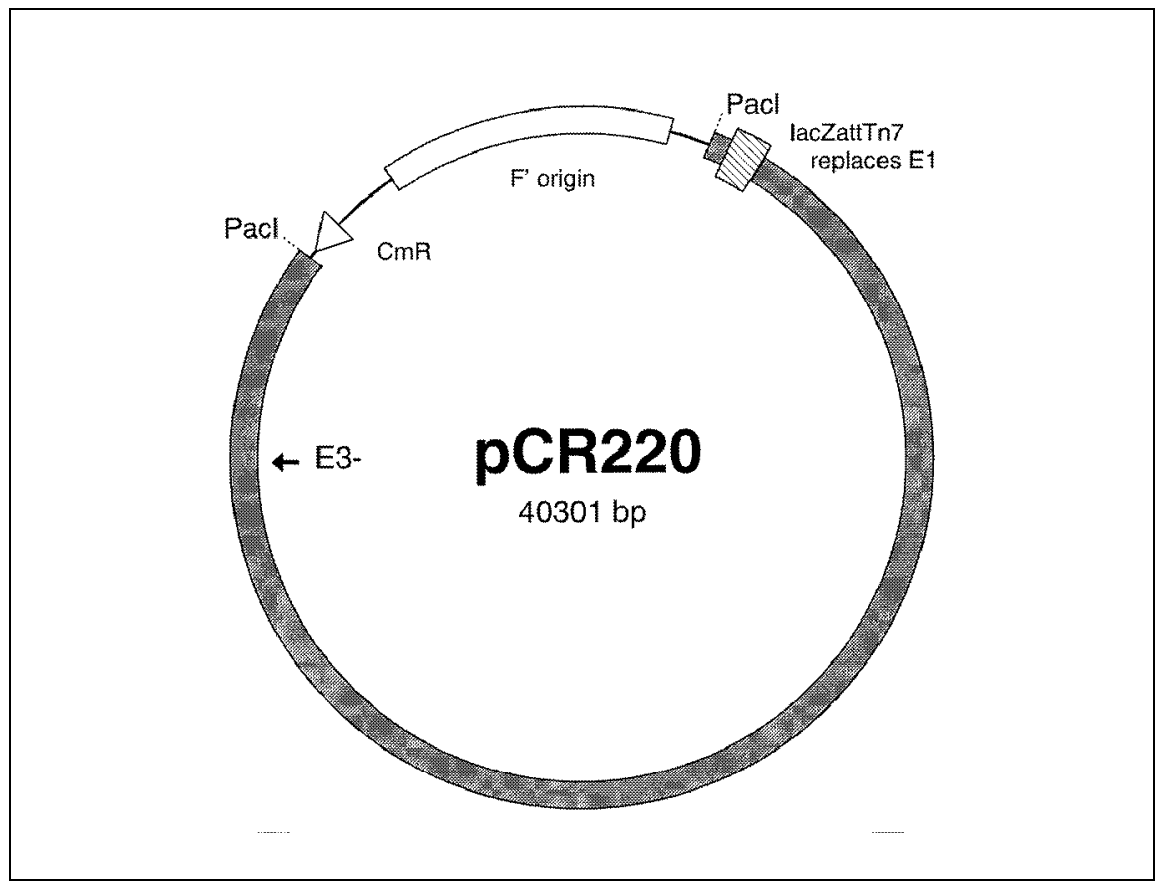

Figure 1. Diagram of admid vector pCR220. The shaded area represents Ad5 sequences and the hatched box indicates the lacZatt Tn7, which replaces approximately $3 \mathrm{~kb}$ from the Ad5 E1 region. Indicated are the $\mathrm{F}^{\prime}$ origin of replication (open box) and $\mathrm{Cm}^{\mathrm{R}}$ marker (open arrow). An earlier vector, pCR213, has the same Ad genome with a 2.9-kb PacI (left end of Ad sequences), SwaI (right end of Ad sequences) fragment containing the ColE1 origin and $\beta$-lactamase gene replacing the $6.5-\mathrm{kb} P a c \mathrm{I}$ fragment of pCR220. 
A.

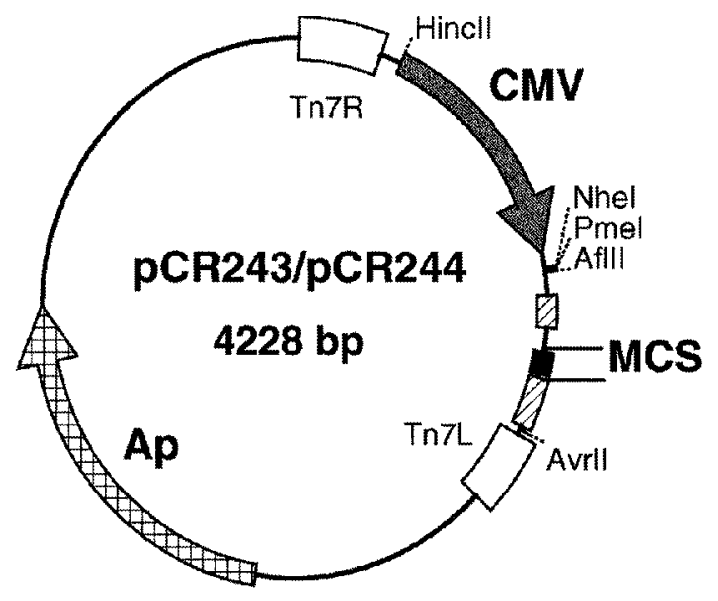

B.

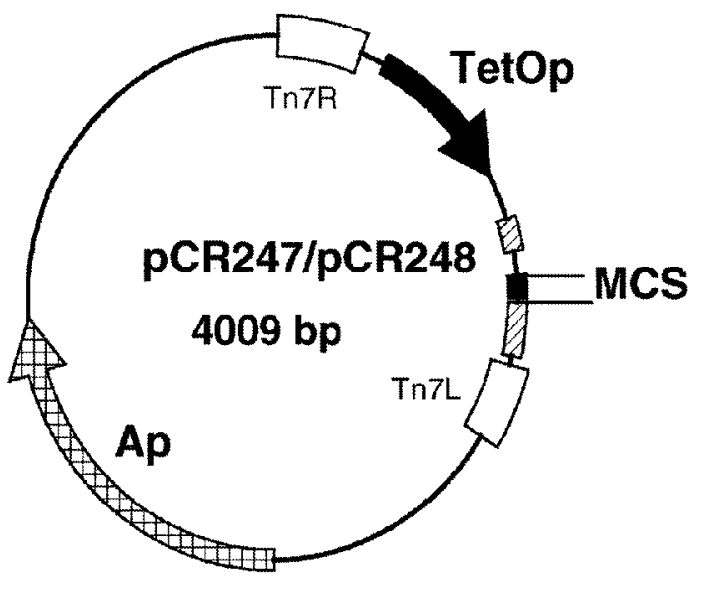

Figure 2. Diagram of transfer vectors. The ColE1 origin, $\mathrm{Ap}^{\mathrm{R}}$ marker, Tn7R and Tn7L, are derived from the 3.1-kb $S a c \mathrm{II}, B c l$ fragment of pFASTBAC1. (A) The 1.0-kb CMV expression cassette has a multiple cloning site (MCS) between the simian virus 40 (SV40) splice and polyadenylation signals. (B) Vectors with the Tc-regulatable promoter were made by replacing the CMV promoter with the 474-bp XhoI, XbaI fragment from pUHG10-3 (9). Transfer vectors containing the luciferase reporter gene, pCR219 and pCR232, were made by inserting the 1.7-kb HindIII, XbaI fragment of pGL3-basic into the MCS of the CMV and TetOp vectors, respectively. Legend: Open boxes, left and right ends of Tn7; hatched filled arrow, Ap ${ }^{\mathrm{R}}$ gene; small and large boxes with left slant, the splice site and polyadenylation signal from SV40, respectively; shaded arrow, CMV promoter; solid arrow, Tc-regulated promoter; filled box MCS-pCR243 and pCR247:PstI/KpnI/NotI/HindIII/NruI/BamHI; pCR244 and pCR248: PstI/KpnI/BamHI/NruI/HindIII/NotI. (The KpnI site is not unique in the TetOp vectors because of the presence of a $\mathrm{KpnI}$ site in the TetOp promoter.)

5'-GGTCGAGCGTCTTCGAAGCGC$3^{\prime}$ and priCR2 5'-CCGTCTTCGAACCAATCAGCAAACC-3') replaces about $3 \mathrm{~kb}$ of the E1A/E1B region of Ad5 (bases 356-3329 deleted; GenBank ${ }^{\circledR}$ accession no. M73260). The final construct, pCR220, is maintained as a low copy number plasmid in E. coli, and produces a $\beta$-gal ${ }^{+}$phenotype on media containing Bluo-gal and IPTG. Restriction of pCR220 with PacI produces two linear DNA fragments. One fragment is essentially an $\mathrm{E}^{-}, \mathrm{E}^{-}$recombinant Ad genome with lacZatt Tn7 replacing E1. The other fragment carries the DNA needed for replication and selection in E. coli.

Although functionally deleted for $\mathrm{E} 3$, the E3 mutation in pCR220 is derived from $d l 309$, in which the deleted Ad5 E3 DNA was replaced with heterologous DNA, which results in little increase in the cloning capacity (5). To accommodate larger insert sizes, it was desirable to remake the E3 mutation as a physical deletion of DNA. This was accomplished using standard DNA cloning techniques to replace the $\mathrm{dl309}$ derived E3 mutation with an E3 mutation, which was made by deleting the 1.9-kb $X b a \mathrm{I}$ fragment from wild-type Ad5 to create pCR249. The Ad genomes of pCR249 and pCR220 are 32.0 $\mathrm{kb}$ and $33.8 \mathrm{~kb}$, respectively, allowing insertions up to $5.8 \mathrm{~kb}$ in pCR249 and $4.0 \mathrm{~kb}$ in pCR220 without exceeding the Ad packaging capacity (6).

Plasmid transfer vectors containing mini-Tn7 elements (Figure 2) were constructed using standard cloning techniques. Further details on the constructions of homing and transfer vectors are available from the authors.

\section{Construction of $E$. coli Strains DH10B (Admid220) and DH10B (Admid249)}

DH10B competent cells were transformed with extrachromosomal DNA isolated from DH10Bac and selected on $\mathrm{Tc} . \mathrm{Tc}^{\mathrm{R}}, \mathrm{Ap}^{\mathrm{S}}$ kanamycin-sensitive colonies containing pMON7124 were isolated. Competent DH10B (pMON7124) cells were transformed with either pCR220 or pCR249. The transformants were plated on LB agar $+\mathrm{Tc}+$ $\mathrm{Cm}+$ Bluo-gal + IPTG and were designated DH10B (Admid220) and DH10B (Admid249), respectively. 
Table 1. Yield of Recombinant Ad from 293 Transfection

\begin{tabular}{|c|c|c|c|}
\hline DNA & Treatment & $\begin{array}{l}\text { Location of restriction } \\
\text { enzyme site }\end{array}$ & FFUb \\
\hline pCR220::Tn219 & Pacl & left, right & $5.7 \times 10^{7}$ \\
\hline $\begin{array}{l}\text { pCR220::Tn219/ } \\
\text { pMON7124/pCR219 }\end{array}$ & Pacl & left, right & $7.7 \times 10^{5}$ \\
\hline pCR220::Tn232 & Pacl & left, right & $3.4 \times 10^{7}$ \\
\hline $\begin{array}{l}\text { pCR220::Tn232/ } \\
\text { pMON7124/pCR232 }\end{array}$ & Pacl & left, right & $8.5 \times 10^{5}$ \\
\hline pCR213 & none & none & $7.6 \times 10^{4}$ \\
\hline pCR213 & Pacl & left & $6.8 \times 10^{4}$ \\
\hline pCR213 & Swal & right & $2.7 \times 10^{7}$ \\
\hline pCR213 & Pacl, Swal & left, right & $1.1 \times 10^{7}$ \\
\hline \multicolumn{4}{|c|}{ arelative to the ends of the Ad genome } \\
\hline \multicolumn{4}{|c|}{$\begin{array}{l}\text { b60-mm dishes of } 293 \text { cells were transfected with } 2 \mu \mathrm{g} \text { DNA using } 40 \mu \mathrm{g} \\
\text { LIPOFECTAMINE; five days later, monolayers were scraped from the dishes in } 4 \\
\mathrm{~mL} \text { media, the suspension was frozen and thawed three times and titered on } 293 \\
\text { monolayers. }\end{array}$} \\
\hline
\end{tabular}

\section{Transposition of Mini-Tn7 Elements from Transfer Vectors into Admids}

Transposition was performed by transforming $80 \mu \mathrm{L}$ competent DH10B (Admid220) with $10 \mathrm{ng}$ uncut transfer vector. The competent cells were mixed with the transfer vector and incubated for $20 \mathrm{~min}$ on ice. After a 45-s heat shock at $42^{\circ} \mathrm{C}$ and a subsequent 2-min incubation on ice, $900 \mu \mathrm{L}$ SOC media (Life Technologies) were added, and the cells were grown for $4 \mathrm{~h}$ at $37^{\circ} \mathrm{C}$ with aeration. The cultures were then plated on $\mathrm{LB}$ agar $+\mathrm{Cm}+\mathrm{Tc}+\mathrm{Ap}+$ Bluo-gal + IPTG and grown for $24 \mathrm{~h}$ at $37^{\circ} \mathrm{C}$. The $\beta$-gal ${ }^{-}$transformants were verified as transposition products by PCR (one primer binding to the Ad sequences of the admid, the other primer binding to an sequence on the mini-Tn7 of the transfer vector). Some of the transposed constructs were further verified using restriction enzyme digests after segre- 


\section{Research Report}

gating the low copy number recombinant admid from the high copy number pMON7124 helper plasmid and the transfer vector by $\mathrm{CaCl}_{2}$ transformation.

\section{Production and Purification of Ad}

One day after plating about $2 \times 10^{6}$ HEK 293 cells per $60-\mathrm{mm}$ dish, the cells were transfected using $2 \mu \mathrm{g}$ plasmid DNA and $40 \mu \mathrm{g}$ LIPOFECTAMINETM (Life Technologies). The DNA/LIPOFECTAMINE complex was incubated with the cells for $6 \mathrm{~h}$ in serum-free media before diluting the transfection mixture with an equal volume of media containing $4 \%$ bovine calf serum. The cells were harvested after six days, usually before any cytopathic effect was observed. The cells were frozen and thawed three times, the cell debris pelleted and the virus-containing supernatant was titered and amplified.

The virus was titered by infecting confluent plates of HEK 293 cells with varying virus dilutions for $1 \mathrm{~h}$. Eighteen

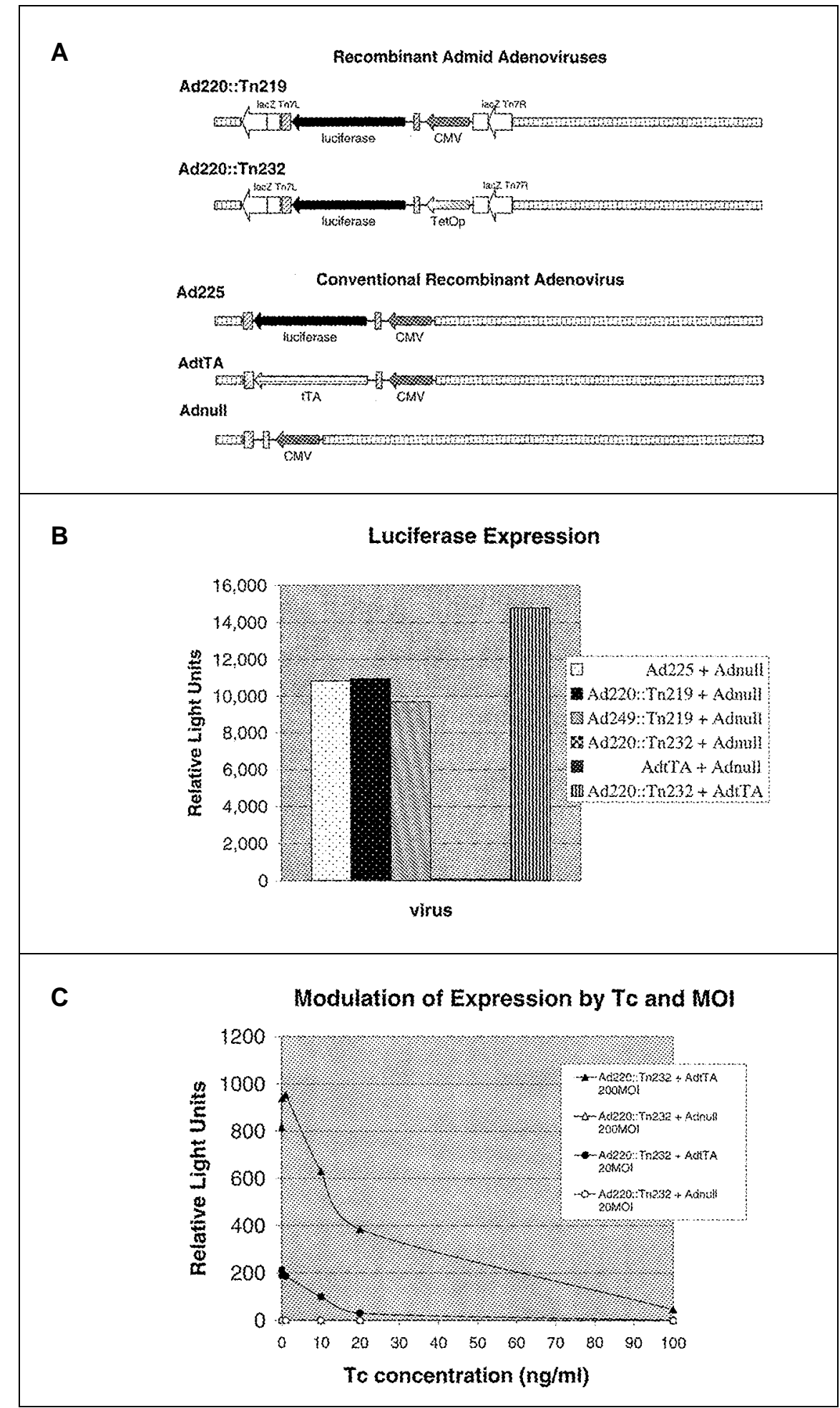

Figure 3. Luciferase expression from recombinant Ads. (A) diagram of recombinant Ads. Viruses that were made by the admid system have lac $\mathrm{Z}$ and $\mathrm{Tn} 7$ sequences (open symbols) flanking the luciferase expression cassette. Hatched boxes: Ad5 sequences; open arrow: lacZatt $\operatorname{Tn} 7$; open boxes: Tn7L and Tn7R; filled arrow: luciferase coding sequence; shaded arrow: CMV immediate early promoter; hatched arrow: Tc-regulated promoter; boxes with left slant fill: SV40 splice (small box) and SV40 polyadenylation signal (large box). (B). Luciferase expression $48 \mathrm{~h}$ after infecting IMR-90 cells at an MOI of 100 (50 MOI luciferase virus $+50 \mathrm{MOI}$ either Adnull or AdtTA virus). (C) Tc-regulated expression $48 \mathrm{~h}$ after infecting IMR-90 cells at an MOI of 200 or 20 (half of virus contained luciferase gene and half of virus either Adnull or AdtTA). When indicated, Tc was added one day before viral infection. 
hours later, the cells were fixed with $90 \%$ methanol, and the number of infected cells was determined by fluorescent microscopy [primary antibody: mouse $\mathrm{Ab}$ to Ad (Biodesign International, Kennebunk, ME, USA); secondary antibody: FITC-labeled goat anti-mouse antibody (ICN, Aurora, OH, USA)]. Amplified virus was extracted with Freon and purified by banding in $\mathrm{CsCl}$ gradients (12). The purified virus was dialyzed against $10 \mathrm{mM}$ Tris $\mathrm{HCl}(\mathrm{pH} 8.0)$, $10 \mathrm{mM} \mathrm{MgCl}_{2}, 150 \mathrm{mM} \mathrm{NaCl}, 10 \%$ glycerol and stored at $-25^{\circ} \mathrm{C}$.

\section{Construction of Conventional Control Viruses}

The control viruses, Adnull and Ad225, were constructed by the traditional method of homologous recombination in 293 cells (4). The recombinant viruses were identified by PCR analysis of the primary amplification of individual plaques, purified again and then amplified. The viruses were produced and purified as described for the viruses produced using the admid system.

\section{Infection of Cell Lines and Luciferase Assays}

IMR-90 cells were plated at $5 \times 10^{4}$ cells/well in 6-well dishes. Two days later, after undergoing two population doublings, triplicate wells were infected with Ad mixtures at the indicated MOI. Each infection contained equal amounts of luciferase virus and either Adnull or AdtTA virus. Two days later, the cells were harvested and assayed for luciferase activity using the luciferase assay system with cell culture lysis reagent as recommended by the supplier (Promega). Luciferase activity was quantitated using a ML3000 luminometer (Dynatech Labs, Chantilly, VA, USA). Where indicated, the cell culture medium was supplemented with Tc starting one day before viral infection.

\section{RESULTS AND DISCUSSION}

\section{Construction of Plasmid Vectors}

The development of the admid system required the construction of two plasmid components: (i) a low copy number admid homing vector containing an Ad genome with a lacZattTn7 site (Figure 1), and (ii) an admid transfer vector with a mini-Tn7 containing an expression cassette appropriate for expression in Ad vectors (Figure 2). The target site for $\operatorname{Tn} 7$ insertion, att $\operatorname{Tn} 7$, is located in the lac Z gene. Transposition into attTn7 disrupts the lac $Z$ reading frame, resulting in a $\beta-\mathrm{gal}^{-}$phenotype. The final construct is maintained as a low copy number plasmid in $E$. coli to facilitate screening for the $\beta$-gal ${ }^{-}$phenotype.

The mini-Tn7 transposition cassette within the admid transfer vectors was designed to be as small as possible while providing efficient transposition, screening for transposed admids and transgene expression in mammalian cells. The "bacmid" system described by Luckow et al. (22) incorporates a gentamycin-resistance marker within the mini-Tn7 to aid in the selection of transposed constructs. We have found the antibiotic marker within the $\mathrm{Tn} 7$ transposition cassette to be unnecessary; similar transposition efficiencies were obtained whether or not an antibiotic marker within the mini-Tn7 cassette was used to select for the transposed constructs (data not shown). Several mini-Tn7 admid transfer vectors have been constructed (Figure 2). Convenient restriction sites are present in which to insert sequences under the control of the cytomegalovirus (CMV) or Tc-regulatable promoter. These sites may also be used to replace the CMV promoter with any other desired regulatory sequence.

\section{Transposition and Confirmation of Transposition by PCR}

The host for transposition was an $E$. coli strain DH10B that contained the plasmid pMON7124 [encoding the trans-acting $\operatorname{Tn} 7$ transposase genes and Tc-resistance (3)] and an admid (containing the lacZatt $\operatorname{Tn} 7$ gene). Transposition occurs following the introduction of a transfer vector containing a miniTn7 element into the host strain. The transposition and screening for recombinant admids containing a mini-Tn7 expression cassette occurs in a single step that is efficient and easily scored as a $\beta$-gal- phenotype on media containing Bluo-gal and IPTG. The percentage of $\beta$-gal ${ }^{-}$colonies obtained appears to 
vary based on an uncharacterized feature of the competent DH10B admid cells used for the transposition. For example, the nine transpositions performed with the first batch of frozen competent cells were carefully monitored. The transposition efficiency averaged $23 \%$, range $9 \%-40 \% \beta$-gal $^{-}$ colonies. Although more recent batches of frozen competent cells have been less efficient, our experience using this system to make over 40 recombinant Ads has been that every transposition experiment has yielded $\beta$-gal- colonies. Furthermore, all the $\beta$-gal ${ }^{-}$colonies we have tested represent true transposition products (PCR product using one primer binding to sequences present on the admid and the second primer binding to the transfer vector).

\section{Transfection of HEK 293 Cells and Initial Viral Titers}

After clonal isolation of the $\beta$-gal ${ }^{-}$ isolates, admid DNA was isolated and restricted with restriction enzyme PacI.
Transfection of HEK 293 cells with admid DNA led to efficient production of infectious Ad (Table 1). Higher amounts of virus were produced if the cells were transfected with only the transposed recombinant admid DNA rather than a mixture of DNAs containing admid, pMON7124, and transfer vector. This likely reflects the increased molar amount of admid DNA present in $2 \mu \mathrm{g}$ pure admid DNA versus the lesser molar amount of admid DNA in $2 \mu \mathrm{g}$ mixed DNA (a low copy number admid and two high copy number plasmids).

Results obtained with pCR213, a predecessor of pCR220 that contains a PacI site at the left end and a $S w a I$ site at the right end of the Ad genome (see Figure 1 legend), demonstrated that the virus was produced even if the admid DNA was not restricted before transfecting 293 cells. However, approximately 100fold more virus was obtained from admid DNA that was restricted at either the right or both ends of the adenoviral genome compared to undigested admid DNA or DNA restricted only at the left end of their Ad genome (Table 1). Therefore, in most cases, it will be beneficial to digest the admid with $P a c$ I before transfection. However, in those rare cases when the expression cassette has a recognition site for PacI, the digestion can be omitted if an additional round of amplification is performed before largescale virus production.

\section{Titers and Expression from Admid Vectors}

We have also compared the yield of infectious virus and expression capabilities of E1-, E3- Ads generated by conventional and admid methods. Both the traditional method of homologous recombination in 293 cells and the admid method were used to construct viruses expressing luciferase. The CMV-luciferase expression cassette is identical between Ad225 and Ad220::Tn219. The only differences between Ad225 and $\operatorname{Ad} 220:: \operatorname{Tn} 219$ are the addition of lac $\mathrm{Z}$ and $\mathrm{Tn} 7$ sequences flanking both sides of the CMV-luciferase expression cassette in Ad220::Tn219 (Figure 3A). When equivalent numbers of 293 cells were infected and the virus was purified, similar amounts of virus were obtained [Ad225, 9.2 $\times 10^{10}$ focus-form- ing units (FFU); Ad220::Tn219, $6.5 \times$ 1010; Ad249::Tn219, 6.6 × 1010 FFU]. Neither the titer nor the final yield of virus was altered by the lacZ or Tn7 sequences of $\operatorname{Ad} 220:: \operatorname{Tn} 219$ or the alternative $\mathrm{E} 3$ deletion of pCR249.

To test the expression levels obtained from each virus, IMR-90 cells were infected with recombinant Ad, and the luciferase activity was quantitated (Figure 3B). A comparison of the luciferase activity from cells infected with either Ad225 or Ad220::Tn219 shows the same luciferase expression from viruses made by either method. This indicates that the flanking lac $\mathrm{Z}$ and $\mathrm{Tn} 7$ sequences do not affect the expression of the CMV promoter. Essentially equal expression was also obtained from Ad249::Tn219 indicating the alternative $\mathrm{E} 3$ deletion also did not affect luciferase expression.

\section{Ads for Efficient Functional Analysis}

The ability to modulate the level of expression of an introduced gene over a broad range should facilitate efficient functional analysis experiments by allowing such studies to be carried out at very high, very low or physiological levels of gene expression with one set of viral reagents. Figure $3 \mathrm{C}$ demonstrates the broad range of expression that can be obtained using the Tc-regulated gene expression system (10) in Ad vectors. Little to no expression is detected in cells infected with the tetOp-luciferase virus (Ad220::Tn232) in the absence of the AdtTA transactivator virus (open symbols). However, when cells are co-infected with both viruses, a large amount of luciferase activity is observed (filled symbols). Also, adding Tc to the cell culture media allowed for the fine-tuning of the absolute level of luciferase expression.

The admid system could also be adapted to carry out efficient protein expression and gene therapy studies. By moving the promoter from the transfer vector onto the admid in such a way that after transposition the transposed gene is regulated by a promoter flanking the lacZattTn7, it should be possible to make one transfer vector that could be transposed into a series of admids that contain different promot- 
ers. Making similar adaptations to bacmids would allow the same "universal" transfer vector to be used to make both Ads and baculoviruses. It should also be possible to generate a wide range of viral vectors with attTn7 sites (e.g., herpes, AAV and retroviral vectors). If the appropriate homing vectors (that is, admids, bacmids, etc.) are made, a "universal" transfer vector could further increase the speed and lower the costs of expressing large amounts of functional protein for purification, for in vitro and in vivo functional analysis or to optimize delivery for gene therapy. Such increases in efficiency will be required to analyze the many genes being discovered by the human genome project and for rapid, cost-effective comparisons among viral delivery systems.

\section{ACKNOWLEDGMENTS}

We are grateful to D. Hanahan for providing E. coli BJ5183, P. Fasani-
Ghersa for AdtTA and S. Neill, J. Bisi, L. Babiss and J. Hanvey for many helpful discussions.

\section{REFERENCES}

1.Aoki, K., C. Barker, X. Danthinne, M.J. Imperiale and G.J. Nabel. 1999. Efficient generation of recombinant adenoviral vectors by Cre-lox recombination in vitro. Mol. Med. 5:224-231.

2.Ausubel, F.M., R. Brent, R.E. Kingston, D.D. Moore, J.G. Seidman, J.A. Smith and K. Struhl (Eds.). 1995. Current Protocols in Molecular Biology. John Wiley \& Sons, New York.

3.Barry, G.F. 1988. A broad-host-range shuttle system for gene insertion into the chromosomes of gram-negative bacteria. Gene 71:7584.

4.Berkner, K.L. 1988. Development of adenovirus vectors for the expression of heterologous genes. BioTechniques 6:616-629.

5.Bett, A.J., V. Krougliak and F.G. Graham. 1995. DNA sequence of the deletion/insertion in early region 3 of Ad5 dl309. Virus Res. 39:75-82.

6.Bett, A.J., L. Prevec and F.L. Graham. 1993. Packaging capacity and stability of human adenovirus type 5 vectors. J. Virol. 67:5911-5921.

7.Chartier, C., E. Degryse, A. Dieterle, A. Pavirani and M. Mehtali. 1996. Efficient generation of recombinant adeovirus vectors by homologous recombination in Escherichia coli. J. Virol. 70:4805-4810.

8.Crouzet, J., L. Naudin, C. Orsini, E. Vigne, L. Ferrero, A. Le Roux, P. Benoit, M. Latta et al. 1997. Recombinational construction in Escherichia coli of infectious adenoviral genomes. Proc. Natl. Acad. Sci. USA 94:14141419.

9.Ghersa, P., R. Hooft van Huijsduijnen, J. Whelan, Y. Cambet, R. Pescini and J.F. DeLamarter. 1994. Inhibition of E-selectin gene transcription through a cAMP-dependant protein kinase pathway. J. Biol. Chem. 269: 29129-29137.

10.Gossen, M. and H. Bujard. 1992. Tight control of gene expression in mammalian celss by tetracycline-responsive promoters. Proc. Natl. Acad. Sci. USA 89:5547-5551.

11.Graham, F.L., J. Smiley, W.C. Russell and R. Nairn. 1977. Characteristics of a human cell line transformed by DNA from human adenovirus type 5. J. Gen. Virol. 36:59-72.

12.Greber, U.F., M. Willets, P. Webster and A. Helenius. 1993. Stepwise dismantling of adenovirus 2 during entry into cells. Cell 75:477486.

13.Hanahan, D. 1983. Studies on transformation of Escherichia coli with plasmids. J. Mol. Biol. 166:557-580.

14.He, T.-C., S. Zhou, L.T. da Costa, J. Yu., K.W. Kinzler and B. Vogelstein. 1998. A simplified system for generating recombinant adenoviruses. Proc. Natl. Acad. Sci. USA 95:2509-2514.

15.Hearing, P. and T. Shenk. 1983. The adenovirus type 5 E1A transcriptional control re- gion contains a duplicated enhancer element. Cell 33:695-703.

16.Imler, J.-L. 1995. Adenovirus vectors as recombinant viral vaccines. Vaccine 13:11431151.

17.Ketner, G., F. Spencer, S. Tugendreich, C. Connelly and P. Hieter. 1994. Efficient manipulation of the human adenovirus genome as an infectious yeast artifical chromosome clone. Proc. Natl. Acad. Sci. USA 91:61866190.

18.Kim, U.-J., H. Shizuya, P.J. deJong, B. Birren and M.L. Simon. 1992. Stable propagation of cosmid sized human DNA inserts in an F factor based vector. Nucleic Acids Res. 20:1083-1085.

19.Kozarsky, K.F. and J.M. Wilson. 1993. Gene therapy: adenovirus vectors. Curr. Opin. Genet. Dev. 3:499-503.

20.Lamarche, N., B. Massie, M. Richer, H. Paradis and Y. Langelier. 1990. High level expression in 293 cells of the herpes simplex virus type 2 ribonucleotide reductase subunit 2 using an adenovirus vector. J. Gen. Virol. 71:1785-1792.

21.Lehrman, S. 1999. Virus treatment questioned after gene therapy death. Nature 401: 517-518.

22.Luckow, V.A., S.C. Lee, G.F. Barry and P.O. Olins. 1993. Efficient generation of infectious recombinant baculoviruses by sitespecific transposon-mediated insertion of foreign genes into a baculovirus genome propagated in Escherichia coli. J. Virol. 67:4566-4579.

23.Nichols, W.W., D.G. Murphy, V.J. Cristofalo, L.H. Toji, A.E. Greene and S.A. Dwight. 1977. Characterization of a new human diploid cell strain, IMR-90. Science 196:60-63.

24.Noel, R.J., P.A. Antinozzi, J.D. McGarry and C.B. Newgard. 1997. Engineering of glycerol-stimulated insulin secreation in islet beta cells. Differential metabolic fates of glucose and glycerol provide insight into mechanisms of stimulus-secretion coupling. J. Biol. Chem. 272:18621-18627.

25.Souza, D.W. and D. Armentano. 1999. Novel cloning method for recombinant adenovirus construction in Escherichia coli. BioTechniques 26:502-508.

26.Zhao, H., L. Ivic, J.M. Otaki, M. Hashimoto, K. Mikoshiba and S. Firestein. 1998. Functional expression of a mammalian odorant receptor. Science 279:237-242.

Received 22 November 1999; accepted 15 February 2000.

Address correspondence to:

Cynthia Richards

Glaxo Wellcome Inc.

Five Moore Dr.

Research Triangle Park, NC 27709, USA

e-mail:car39385@glaxowellcome.com 